\title{
Homologous repair deficiencies and current insights in clinical evaluation of PARP inhibitors in prostate cancer
}

\author{
Maximilian Marhold · Thaïs Topakian
}

Received: 8 March 2020 / Accepted: 23 March 2020 / Published online: 15 April 2020

(C) The Author(s) 2020

\begin{abstract}
Summary Homologous repair deficiency is a clinically relevant molecular aberration in prostate cancer. The goal of this short review is to summarize the study landscape of treatments targeting these aberrations through discussion of the most relevant clinical trials. Due to its shortness, this review does not claim to be exhaustive and a major focus is being laid on PARP inhibitors in clinical development for prostate cancer.
\end{abstract}

Keywords Prostate cancer - BRCA1 - BRCA2 - PARP . Homologous repair deficiency $\cdot$ Cancer treatment

$\begin{array}{ll}\text { Abbreviations } \\ \text { ADT } & \text { Androgen deprivation therapy } \\ \text { AR } & \text { Androgen receptor } \\ \text { CRPC } & \text { Castration-resistant prostate cancer } \\ \text { DDR } & \text { DNA damage repair } \\ \text { HRR } & \text { Homologous recombination repair } \\ \text { PARPi } & \text { Poly(ADP-ribose) polymerase inhibitors } \\ \text { PCa } & \text { Prostate cancer } \\ \text { PFS } & \text { Progression-free survival }\end{array}$

\section{Introduction}

Prostate cancer (PCa) is the most frequently diagnosed noncutaneous cancer in men and was ranked third regarding incidence among all cancers in the European Union in 2018 [1]. Although prostate-specific antigen (PSA) screening has facilitated early detection, treatment of advanced metastatic PCa remains challeng-

\author{
Dr. M. Marhold, $\mathrm{PhD}(\bowtie) \cdot$ T. Topakian, MSc \\ Division of Oncology, Department of Medicine I, Medical \\ University of Vienna, Währinger Gürtel 18-20, 1090 Vienna, \\ Austria \\ maximilian.marhold@meduniwien.ac.at \\ T. Topakian, MSc \\ thais.topakian@meduniwien.ac.at
}

ing as the disease is highly heterogeneous, exhibiting strong histopathological, genetic and clinical diversity [2]. Since PCa growth is mainly dependent on androgen receptor (AR) expression, androgen deprivation therapy (ADT) targeting AR or AR ligand binding remains one of the standards of care to date. However, despite initial response, patients often develop resistance to ADT, leading to incurable castration-resistant prostate cancer (CRPC).

The endeavor of profiling the molecular and genetic landscape of PCa and searching for novel predictive biomarkers that specify and optimize therapy has recently led to the discovery of multiple genomic alterations. Next generation sequencing studies of prostate tumors revealed a high number of recurrent gene mutations interfering with homologous recombination repair (HRR). Among others, the Cancer Genome Atlas (TCGA) Research and American Association for Cancer Research networks have identified HRR gene alterations in 19-23\% of metastatic CRPC (mCRPC) samples [3, 4]. Analyzing the TCGA prostate cancer database, Kim et al. found mutations and copy-number variations of DNA damage repair (DDR) genes in $30 \%$ of localized (nonmetastatic) PCa indicating that DDR alterations might occur early during disease progression [5]. Reported prevalence of mutations included HRR genes such as cyclin-dependent kinase 12 (CDK12), RAD51C, breast cancer 1 (BRCA1) or ataxia telangiectasia mutated (ATM). Interestingly, all studies found breast cancer 2 (BRCA2) to be the most commonly mutated gene.

Germline BRCA2 mutations are associated with a high risk of developing cancer across many entities with a recently reported risk of $19-61 \%$ for PCa by age 80 [6]. The concept of "synthetic lethality", initially described in drosophila by Dobzhansky as a state where simultaneous loss-of-function of two genes results in a nonviable phenotype, led to the 
discovery of the synthetically lethal interplay between BRCA1 or BRCA2 with poly(ADP-ribose) polymerase (PARP1). In this case, the DDR deficiencies caused by BRCA1 or 2 loss-of-function lead to cell death if the compensatory DNA repair pathway via PARP1 is inhibited. PARP inhibitors (PARPi) were, thus, developed and approved for the treatment of breast and ovarian cancers. Furthermore, emerging evidence indicated a strong relationship between BRCA1/2 deficiencies with sensitivity to DNA-damage causing platinum-based chemotherapy in breast and ovarian, but also in PCa [7]. A synthetic lethal mode-of-action was also claimed for radium-223 treatment in mCRPC with bone metastasis and homologous repair gene defects (HRD) [8]; however the mechanistic explanation remained most reasonable for PARP, which, as components of base excision repair (BER), restore damaged DNA in case of HRR gene loss.

Beside the synthetic lethality of PARPi in the presence of HRR aberrations, numerous studies highlight further effects of PARPi activity in PCa. Gui et al. demonstrated that selectively targeting PARP2 blocked its interaction with FOXAl and attenuated AR-signaling [9]. Conversely, Li et al. identified an AR and c-myb upregulated HRR gene signature that correlated with castration resistance, metastasis, relapse and reduced survival and demonstrated that targeting AR by enzalutamide led to downregulation of this specific gene set [10]. These findings suggest PARPi as an alternative therapeutic approach for ADT irrespective of HRR status and indicate a potential combinatory effect with antiandrogens such as apaor enzalutamide. Several PARPi have been or are currently evaluated in clinical trials for the treatment of PCa, including olaparib, rucaparib, talazoparib, veliparib and niraparib. While none of them are currently approved within Europe, numerous trials already show promising activity.

The single-arm phase II trial TOPARP-A recruited 50 men with $\mathrm{mCRPC}$, who had previously undergone chemotherapy and/or ADT, for the treatment olaparib irrespective of their HRR status. Response was defined according to RECIST, with a decline in PSA levels by at least $50 \%$ or a reduction of counted circulating tumor cells to less than 5 per $7.5 \mathrm{~mL}$ blood. Of 16 patients who responded to olaparib, $88 \%$ were identified to carry HRR mutations. Within responders, HRR mutation carriers exhibited significantly longer radiologic progression-free survival (PFS, 9.8 vs. 2.7 months). Interestingly, durable responses for more than a year were achieved for all 8 patients with BRCA1/2 aberrations [11].

To further evaluate antitumor activity of olaparib stratified by specific DDR mutations, the TOPARP-B phase II trial analyzed responses of 98 men genomically preselected for putative pathogenic mutations in DDR pathways. All patients had received prior docetaxel and the majority prior enzalutamide or abiraterone. Striking for this pretreated collective were the response rates of 57 and $41 \%$ in the 400 and $300 \mathrm{mg}$ treatment arms, respectively. Again, BRCA1/2-mutated patients represented the subgroup with strongest response and longest median radiographic PFS (8.3 months) [12].

The still ongoing phase III trial PROfound is investigating 387 men with mCRPC and at least one mutation in DDR genes subjected to treatment with olaparib or physician's choice of enzalutamide or abiraterone. Two cohorts were defined based on mutations with BRCA1/2 and ATM (cohort A) and other alterations (cohort B). In an interim analysis presented at ESMO congress last year, olaparib treatment resulted in significantly prolonged radiographic progression-free survival in cohort A (HR 0.34) and the overall population (cohort A+B, HR 0.49) as well as a trend for prolonged overall survival not reaching the previously determined levels of statistical significance for cohort A. Most likely, heavy cross-over between treatment arms hindered the observation of a statistically significant difference in OS for cohort A in the interim analysis (HR 0.64, n.s.), but could be met in the final analysis [13].

Besides olaparib monotherapy, combinatorial effects of olaparib with antihormonal agents are being investigated. Due to its brevity, this short review cannot cover all currently ongoing trials. Of note, however, Clarke et al. assessed the efficacy and tolerability of abiraterone plus olaparib and compared it to abiraterone with placebo. A total of 71 patients were assigned to each cohort, of which 14 and $11 \%$ were identified to have HRR deficiencies in the olaparib and placebo arm, respectively. Radiographic PFS was significantly prolonged in the abiraterone plus olaparib group regardless of HRR status (13.8 vs 8.2 months), again with more adverse effects in the olaparib arm [14].

Another promising PARP inhibitor, talazoparib, is being evaluated for CRPC patients with DDR defects. Described as being the most potent inhibitor in trapping PARP enzymes at single-strand breaks in vitro, talazoparib provides high metabolic stability in mice and is orally bioavailable [15]. The phase II study TALAPRO-1 presently examines efficacy and safety of talazoparib ( $1 \mathrm{mg} /$ day) stratified by DDR mutations of 11 genes. Interim analyses showed highest efficacy of talazoparib treatment for BRCA1/2 and PALB2 deficient CRPC patients with ORR of 50 and 33\%, respectively. Interestingly, the BRCA1/2 cohort had the longest radiographic PFS with 8.2 months compared to patients with other DDR alterations [16, 17]. In addition, the currently ongoing TALAPRO-2 phase III study is examining the combination therapy of talazoparib and enzalutamide. Recently presented intermediate results of TALAPRO-2 indicated that $0.5 \mathrm{mg}$ talazoparib per day in combination with background enzalutamide treatment in therapy-naïve patients had a manageable safety profile and led to a prominent de- 
cline in PSA levels after a median treatment duration of 11 weeks [18].

The high expectations for PARPi efficacy in CRPC treatment are further reflected by 10 currently recruiting clinical trials with the aim to examine rucaparib in combination with other anticancer treatments or as monotherapy. Preliminary results of the phase II study TRITON2 demonstrated high ORR (43.9\%) with durable response over 24 weeks for the majority of mCRPC patients carrying BRCA mutations. However, only poor response could be achieved for patients with other mutations than BRCA [19]. Similar interim results were presented for the GALAHAD phase II study with highest ORR (41\%) and rPFS of 8.2 months for BRCA mutated CRPC patients treated with the PARPi niraparib [20].

Besides combinatorial therapies of PARPi, antihormonal agents and chemotherapy, the clinical research focus has recently been placed on the question whether PARP inhibition together with immune checkpoint inhibition would show antitumor activity. Several preclinical studies confirmed that PARP inhibition triggers STING activation and promotes type I immunity, which may benefit in increased inflammation and tumor immune infiltration [21]. To our knowledge, there are two currently recruiting phase II trials investigating this topic in CRPC. With final results pending, these two studies are currently assessing the combination of PD-L1 inhibitors avelumab with talazoparib and durvalumab with olaparib, respectively. The latter combination was tested in 17 CRPC patients with preliminary response rates of $53 \%$ for those with DDR gene mutations. The treatment increased immune response in some patients by enhancing dendritic cell maturation, $\mathrm{CD} 4^{+}$and $\mathrm{CD} 8^{+}$ T-cell activity, which correlated with prolonged rPFS [22].

\section{Take home message}

PARP inhibitors are promising novel targeting agents for multiple cancer entities. All PARP inhibitors under development have shown significant clinical benefits for BRCA1/2 mutation carriers suffering from prostate cancer and partially for patients with other homologous recombination repair (HRR) defects. Altogether, data from currently ongoing trials are very likely to change the landscape and treatment standard of HRR-deficient prostate cancer and make DNA-damage repair gene mutation status testing required for all prostate cancer patients in the future.

Funding Open access funding provided by Medical University of Vienna.

Conflict of interest M. Marhold and T. Topakian declare that they have no competing interests.
Open Access This article is licensed under a Creative Commons Attribution 4.0 International License, which permits use, sharing, adaptation, distribution and reproduction in any medium or format, as long as you give appropriate credit to the original author(s) and the source, provide a link to the Creative Commons licence, and indicate if changes were made. The images or other third party material in this article are included in the article's Creative Commons licence, unless indicated otherwise in a credit line to the material. If material is not included in the article's Creative Commons licence and your intended use is not permitted by statutory regulation or exceeds the permitted use, you will need to obtain permission directly from the copyright holder. To view a copy of this licence, visit http://creativecommons.org/licenses/by/4.0/.

\section{References}

1. Ferlay J, et al. Cancer incidence and mortality patterns in Europe: Estimates for 40 countries and 25 major cancers in 2018. Eur J Cancer. 2018;103:356-87.

2. Wyatt AW, et al. The diverse heterogeneity of molecular alterations in prostate cancer identified through nextgeneration sequencing. Asian JAndrol. 2013;15(3):301-8.

3. Cancer Genome Atlas Research. The molecular taxonomy of primary prostate cancer. Cell. 2015;163(4):1011-25.

4. Robinson D, et al. Integrative clinical genomics of advanced prostate cancer. Cell. 2015;162(2):454.

5. Kim IE Jr., et al. Similar incidence of DNA damage response pathway alterations between clinically localized and metastatic prostate cancer. BMC Urol. 2019;19(1):33.

6. Lecarpentier J, etal. Prediction ofbreastand prostatecancer risks in male BRCA1 and BRCA2 mutation carriers using polygenic risk scores. JClin Oncol. 2017;35(20):2240-50.

7. Pomerantz MM, et al. The association between germline BRCA2 variants and sensitivity to platinumbased chemotherapy among men with metastatic prostate cancer. Cancer. 2017;123(18):3532-9.

8. Isaacsson Velho P, et al. Efficacy of radium-223 in bonemetastatic castration-resistant prostate cancer with and without homologous repair gene defects. Eur Urol. 2019;76(2):170-6.

9. Gui B, et al. Selective targeting of PARP-2 inhibits androgen receptor signaling and prostate cancer growth through disruption of FOXAl function. Proc Natl Acad Sci U S A. 2019;116(29):14573-82.

10. Li L, et al. Androgen receptor inhibitor-induced "BRCAness" and PARP inhibition are synthetically lethal for castration-resistant prostate cancer. Sci Signal. 2017. https://doi.org/10.1126/scisignal.aam7479.

11. MateoJ, etal. DNA-repair defects and olaparibin metastatic prostate cancer. NEngl J Med. 2015;373(18):1697-708.

12. Mateo J, et al. Olaparib in patients with metastatic castration-resistant prostate cancer with DNA repair gene aberrations (TOPARP-B): a multicentre, open-label, randomised, phase 2 trial. Lancet Oncol. 2020;21(1):162-74.

13. Hussain M, Mateo J, Fizazi K, Saad F, Shore ND, Sandhu S, et al. PRPROfound:phase III study of olaparib versus enzalutamide or abiraterone for metastatic castration-resistant prostate cancer (mCRPC) with homologous recombination repair(HRR) gene alterations. Ann Oncol. 2019;30 (suppl_5): v851-v934. https://doi.org/10.1093/annonc/ mdz394.

14. Clarke N, et al. Olaparib combined with abiraterone in patients with metastatic castration-resistant prostate cancer: a randomised, double-blind, placebo-controlled, phase 2 trial. Lancet Oncol. 2018;19(7):975-86. 


\section{short review}

15. Shen Y, et al. BMN 673, a novel and highly potent PARP1/2 inhibitor for the treatment of human cancers with DNA repair deficiency. Clin Cancer Res. 2013;19(18):5003-15.

16. de Bono J, Higano CS, Saad F, et al. TALAPRO-1: An openlabel, response rate phase II study of talazoparib (TALA) in men with DNA damage repair (DDR) defects and metastatic castration-resistant prostate cancer (mCRPC) who previously received taxane-based chemotherapy (CT) and progressed on greater than or equal to one novel hormonal therapy (NHT). JClin Oncol. 2019;37(7_suppl):342-342.

17. De Bono J. ASCO GU 2020: a phase II study of Talazoparib in men with DNA damage repair mutations and metastatic castration-resistant prostate cancer presented at the 2020 genitourinary cancers symposium. ASCO GU \#GU20; 13-15. February 2020; San Francisco. Int. J. Clin. Oncol. 2019;38:6_suppl, 119.

18. ASCO. Clinical and safety outcomes of TALAPRO-2: a twopart phase III study of talazoparib in combination with enzalutamide in metastatic castration-resistant prostate cancer. 2019 ASCO Annual Meeting \#ASCO19; 31. May-4. June; Chicago. 2019.

19. AbidaW.ESMO2019: preliminaryresultsfrom the TRITON2 study of Rucaparib in patients with DNA damage repairdeficient mCRPC: updated analyses presented at the 2019 European society for medical oncology annual meeting. ESMO 2019 \#ESMO19; 27 Sept-1 Oct 2019; Barcelona. https://doi.org/10.1093/annonc/mdz248.003.
20. Smith MR, Sandhu SK, Kelly WK, et al. Phase II study of niraparib in patients with metastatic castration-resistant prostate cancer (mCRPC) and biallelic DNA-repair gene defects (DRD): Preliminary results of GALAHAD. J Clin Oncol. 2019;37:202-202.

21. Hartlova A, et al. DNA damage primes the type I interferon system via the cytosolic DNA sensor STING to promote antimicrobial innate immunity. Immunity. 2015;42(2):332-43.

22. Karzai F, et al. Activity of durvalumab plus olaparib in metastatic castration-resistant prostate cancer in men with and without DNA damage repair mutations. J Immunother Cancer. 2018;6(1):141.

Publisher's Note Springer Nature remains neutral with regard to jurisdictional claims in published maps and institutional affiliations.

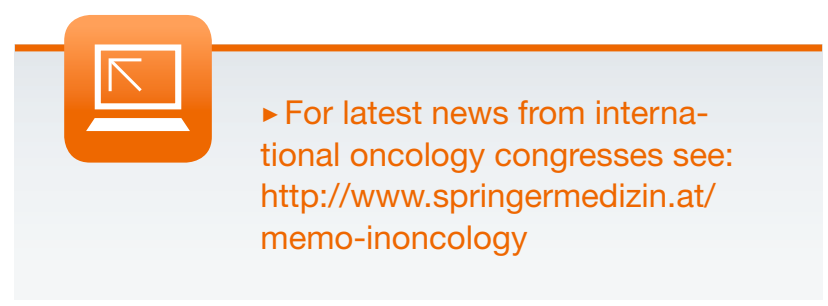

